\title{
Spinal Cord Infarct During Birth
}

National Cancer Institute

\section{Source}

National Cancer Institute. Spinal Cord Infarct During Birth. NCI Thesaurus. Code C101272.

Ischemic necrosis of the spinal cord due to spinal artery occlusion during the birthing process. 